\title{
Six-month visual outcomes for the correction of presbyopia using a small-aperture corneal inlay: single-site experience
}

This article was published in the following Dove Press journal:

Clinical Ophthalmology

2 November 2016

Number of times this article has been viewed

\author{
Majid Moshirfar ${ }^{1,2}$ \\ Tyler S Quist ${ }^{3}$ \\ David F Skanchy 4 \\ Ryan TWallace ${ }^{5}$ \\ Steven H Linn' \\ Phillip C Hoopes Jr' \\ 'Hoopes, Durrie, Rivera Research \\ Center, Hoopes Vision, Draper, \\ ${ }^{2}$ Department of Ophthalmology and \\ Visual Sciences, John A Moran Eye \\ Center, University of Utah School of \\ Medicine, Salt Lake City, ${ }^{3}$ University \\ of Utah School of Medicine, Salt \\ Lake City, UT, ${ }^{4}$ McGovern Medical \\ School, The University of Texas \\ Health Science Center at Houston, \\ TX, ${ }^{5}$ Brigham Young University, Provo, \\ UT, USA
}

Correspondence: Majid Moshirfar HDR Research Center, Hoopes Vision, I I 820 S State Street, Suite \#200,

Draper, UT 84020, USA

$\mathrm{Tel}+\mathrm{I} 8015680200$

Fax + I 80I 5630200

Email cornea2020@me.com
Objective: The objective of this study was to describe 6-month postoperative efficacy and safety outcomes after monocular KAMRA corneal inlay implantation in emmetropic presbyopic patients.

Study design: This study followed a retrospective chart analysis.

Setting: This study was performed at Hoopes Vision in Draper, UT, USA.

Subjects and methods: Fifty-seven patients met the inclusion criteria of this study and underwent KAMRA corneal inlay implantation following the approval of the United States Food and Drug Association between May 2015 and April 2016 at a single site. Surgery involved femtosecond laser-created corneal pockets of various depths. Efficacy, safety, and patient satisfaction reports were analyzed at 3 and 6 months.

Results: At 6 months follow-up, the monocular uncorrected near visual acuity (UNVA) was Jaeger (J) 4 (20/32), the mean uncorrected distance visual acuity was 20/25, and the mean corrected distance visual acuity was 20/20. At 6 months, $71 \%$ of patients with a pocket depth of $\geq 250 \mu \mathrm{m}$ had a UNVA of $20 / 20$ or better, whereas only $22 \%$ of patients with a shallow pocket depth of $<250 \mu \mathrm{m}$ had a UNVA of $20 / 20$ or better. There was no statistical difference in UNVA at 6 months between virgin eyes and post-LASIK eyes. One patient had an explant and five patients underwent inlay recentration, all of which resulted in improved visual acuity. At 6 months, $72 \%$ of patients reported some level of satisfaction, $26 \%$ of patients reported being "not dependent" on reading glasses, and $62 \%$ of patients reported being able to do most things in bright light without reading glasses.

Conclusion: For patients with emmetropic presbyopia, the KAMRA inlay is a viable treatment option resulting in improved UNVA. Increased pocket depth may be associated with better postoperative outcomes. Safety rates are high, while explantation and recentering rates are low. Overall, patient satisfaction of the KAMRA inlay is good.

Keywords: corneal inlay, presbyopia, KAMRA, AcuFocus

\section{Introduction}

Presbyopia is an age-related physiologic condition in which the eye's ability to adapt for near vision becomes compromised. It is estimated that $>1$ billion people worldwide have impaired near vision caused by presbyopia. ${ }^{1-3}$ Traditionally, the management of presbyopia has included reading glasses, contact lenses, and surgical procedures such as monovision laser assisted in situ keratomileusis (LASIK) and conductive keratoplasty. ${ }^{4}$

In recent years, corneal inlays have become a popular treatment option for presbyopia. The KAMRA corneal inlay (AcuFocus, Inc., Irvine, CA, USA) was approved 
by the United States Food and Drug Association (FDA) in April 2015 for the treatment of plano and near-plano presbyopia. The KAMRA inlay is a small disk implanted into the corneal stroma. Utilizing the concept of pinhole optics, it increases the depth of focus. Ideally, it improves near and intermediate visual acuity without altering distance visual acuity. ${ }^{5}$ Recently published literature supports the efficacy of KAMRA inlays in the treatment of presbyopia..$^{5-9}$

In this study, patients who underwent KAMRA corneal inlay implantation for the treatment of presbyopia at our institution were analyzed. All surgeries occurred post FDA approval of the KAMRA corneal inlay. The primary focus of this paper was to evaluate the 6-month efficacy, safety, and patient satisfaction in this patient population.

\section{Patients and methods}

This is a retrospective study of patients who underwent KAMRA corneal inlay surgery for the correction of presbyopia at our institution between May 2015 and April 2016. All patients involved in this study provided written informed consent in accordance with the tenets of the Declaration of Helsinki to having their data used for research purposes. This study was approved by the Hoopes, Durrie, Rivera Internal Review Board. During this time period, there were 106 patients who underwent KAMRA inlay implantation. Seven patients were excluded from our study because they had simultaneous KAMRA inlay and refractive surgery in the same eye, and two patients were excluded because they had scheduled refractive surgeries in the same eye at a future day postoperatively. Forty patients were excluded because they lacked either 3- or 6-month follow-up data. Of the 57 patients included in this study, many had prior treatment to their nondominant eye. Seventy-four percent of $(42 / 57)$ eyes were virgin eyes. Twenty-two percent (13/57) of eyes had LASIK to achieve emmetropia at some time between 1 month and 16 years before KAMRA inlay implantation and $4 \%(2 / 57)$ of eyes had an intraocular lens procedure between 2 and 9 months before KAMRA inlay implantation.

No patients with severe dry eye syndrome, active eye infection or inflammation, keratoconus, abnormal corneal topography, uncontrolled glaucoma, uncontrolled diabetes, or active autoimmune and connective tissue disease underwent surgery.

\section{The small-aperture corneal inlay}

The KAMRA corneal inlay is an opaque annulus (ringshaped device) implanted into the corneal stroma. The device used in this study was the third-generation KAMRA corneal inlay (ACI7000PDT), which has an outer diameter of $3.8 \mathrm{~mm}$, inner diameter of $1.6 \mathrm{~mm}$, and thickness of $5 \mu \mathrm{m}$. It is made of polyvinylidene difluoride formulated with carbon nanoparticles and is microperforated $(8,400$ nutrition holes). The perforations permit transport of nutrients through the implant in order to maintain the health of the cornea. ${ }^{10}$

The technology of the KAMRA inlay is based on the concept of pinhole optics. The device increases the depth of focus by reducing the opening through which light enters the eye. It is intended for implantation in the nondominant eye and functions to improve near vision without significantly compromising distance visual acuity. ${ }^{5}$

\section{Surgical technique}

All surgeries were performed at a single site by the same group of four surgeons. The surgical technique involving the KAMRA corneal inlay has previously been described in detail. ${ }^{10}$

To create a pocket, a femtosecond laser (150-kHz IntraLase iFS; Abbott Medical Optics Inc., Abbott Park, IL, USA) was used using the following settings: channel width of $4.7 \mathrm{~mm}$, depth of 200-280 $\mu \mathrm{m}$, spot and line separation of $4 \mu \mathrm{m}$, energy of $0.55-0.75 \mu \mathrm{J}$, sidecut radius of $5.66 \mathrm{~mm}$, and sidecut angle of $45^{\circ}$. The KAMRA inlay was then positioned in the stromal bed. If the distance between the center of the pupil and the first Purkinje reflex was $<300 \mu \mathrm{m}$, the marker was centered on the Purkinje reflex. The decision to center the KAMRA inlay on the first Purkinje reflex was based on the view that it is the best reference point for the visual axis. ${ }^{11}$ If the distance between the center of the pupil and the Purkinje reflex differed by $>300 \mu \mathrm{m}$, the marker was centered halfway between the two. This decision was in accordance with the recommendations found in the KAMRA Inlay MD Clinical Pearls Quick Reference Guide. ${ }^{12}$

After implantation, topical ofloxacin was prescribed four times daily for 1 week. Topical Pred Forte (prednisolone acetate $1.0 \%$ ) was prescribed four times daily for 1 month, after which topical fluorometholone $(0.1 \%)$ was used on a slow tapering regimen starting with a dose of four times daily. Preservative-free artificial tears were prescribed hourly for the first month, after which bottled artificial tears were used every 2-3 hours for the following 2 months.

\section{Outcomes}

Efficacy of the KAMRA inlay was the primary focus of this study and was analyzed through the comparison of preoperative and postoperative monocular uncorrected near visual acuity (UNVA), uncorrected distance visual acuity (UDVA), and manifest refraction spherical equivalent (MRSE) in the implanted eye at 3 and 6 months. We also evaluated binocular visual acuity. Distance visual acuity 
was measured using a Snellen chart projected from an LCD

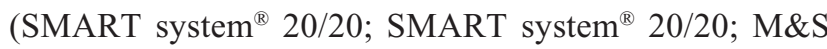
Technologies, Niles, IL, USA) calibrated for both distance and contrast. Near visual acuity was assessed with a near visual acuity chart (Precision Vision ETDRS 2000 Series Chart "2") used at a working distance of $40 \mathrm{~cm}$. The efficacy of the KAMRA inlay was further analyzed through the comparison of preoperative and postoperative monocular UNVA in patients with pocket depths $<250 \mu \mathrm{m}$. and in patients with pocket depths $\geq 250 \mu \mathrm{m}$. Effectiveness of the KAMRA inlay was also evaluated through the comparison of virgin and post-LASIK eyes.

The secondary outcomes in this study included safety and patient satisfaction of the KAMRA inlay. In addition, explantation and repositioning after inlay implantation were addressed. Safety was analyzed through the comparison of preoperative and postoperative corrected distance visual acuity (CDVA) at 3 and 6 months. Patient satisfaction was evaluated through a survey at 3 and 6 months postoperatively, which included rate dependency on reading glasses, overall satisfaction with vision, and the ability to do most (at least 75\%) tasks in "good" light without reading glasses (such as reading text messages, seeing the numbers on the mobile, reading store labels, and viewing the computer screen).

\section{Statistical analysis}

Microsoft Excel 2016 (Microsoft Corporation, Redmond, WA, USA) was used to analyze data and create all tables and graphs displayed in this paper. Our data were nonparametric, and the Wilcoxon rank-sum test was used to determine the significance of difference between unpaired data. The MannWhitney test was applied to determine the significance of difference between unpaired data. A $P$-value $<0.05$ was considered statistically significant.

\section{Results}

\section{Patient characteristics}

This study includes 57 patients. Table 1 shows preoperative patient demographics. Preoperatively, 28\% (16/57) of patients had a UNVA of $20 / 40$ or better, $95 \%$ (54/57) of patients had a UDVA of $20 / 40$ or better, and $100 \%$ (57/57) of patients had a CDVA of 20/20 or better. Preoperative binocular UNVA was $20 / 40$ or better in $42 \%(24 / 57)$ of patients.

\section{Efficacy}

The preoperative and postoperative UNVA in treated eyes is shown in Figure 1. At 3 months postoperatively, the mean UNVA in the implanted eye was Jaeger (J) 4 (20/32 \pm 0.15$)$ and $77 \%(44 / 57)$ of patients had a UNVA of $20 / 40$ or better. At
Table I Patient demographics $(n=57)$

\begin{tabular}{ll}
\hline Age (years) & \\
Mean & $54 \pm 4.09$ \\
$\quad$ Range & 48,65 \\
Sex (n, \%) & \\
$\quad$ Male & $26,45.6 \%$ \\
$\quad$ Female & $31,55.4 \%$ \\
Eye with inlay (n, \%) & $14,24.6 \%$ \\
$\quad$ Right & $43,75.4 \%$ \\
$\quad$ Left & \\
Spherical equivalent (D) & $-0.52 \pm 0.32$ \\
$\quad$ Mean \pm SD & $-1.25,0.13$ \\
$\quad$ Range & \\
UNVA, monocular (lines) & $20 / 50 \pm 0.15$ \\
$\quad$ Mean \pm SD & $20 / 100,20 / 25$ \\
$\quad$ Range & \\
UDVA, monocular (lines) & $20 / 25 \pm 0.15$ \\
$\quad$ Mean \pm SD & $20 / 60,20 / 15$ \\
$\quad$ Range & \\
CDVA, monocular (lines) & $20 / 20 \pm 0.03$ \\
$\quad$ Mean \pm SD & $20 / 20,20 / 15$ \\
Range &
\end{tabular}

Abbreviations: CDVA, corrected distance visual acuity; SD, standard deviation; UDVA, uncorrected distance visual acuity; UNVA, uncorrected near visual acuity.

6 months postoperatively, the mean UNVA in the implanted

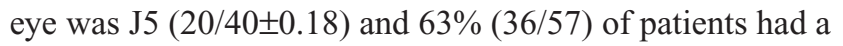
UNVA of 20/40 or better. At 6 months, the improvement in UNVA from preoperative UNVA was statistically significant $(P<0.001)$. Six-month binocular UNVA was $20 / 40$ or better in $77 \%(44 / 57)$ of patients.

The preoperative and postoperative UDVA in treated eyes is shown in Figure 2. At 3 months postoperatively, the mean UDVA in the implanted eye was 20/25 \pm 0.16 and $89 \%$ $(51 / 57)$ of patients had a UDVA of $20 / 40$ or better. At 6 months postoperatively, the mean UDVA in the implanted eye was $20 / 25 \pm 0.15$ and $95 \%(54 / 57)$ of patients had a UDVA of 20/40 or better. At 6 months, there was no statistically significant difference in UDVA from preoperative UDVA $(P=0.30)$.

The mean spherical equivalent refraction was $-0.10 \pm 0.89$ standard deviation (SD) (range: $-2,+1.75$ ) at 6 months with a mean change of $-0.42 \pm 0.76 \mathrm{SD}$ (range: $-1,+2.25$ ) from preoperative data.

\section{Pocket depth comparison}

We categorized a pocket depth of $<250 \mu \mathrm{m}$ as shallow and a pocket depth of $\geq 250 \mu \mathrm{m}$ to be deep. The distribution of pocket depth is shown in Figure 3. The shallow pocket group had a median depth of $210 \mu \mathrm{m}$ with a range of 200-230 $\mu \mathrm{m}$. The deep pocket group had a median depth of $255 \mu \mathrm{m}$ with a range of 250-280 $\mu \mathrm{m}$. Preoperatively, there was no statistical difference between the deep and shallow pocket groups in either UNVA $(P=0.41)$ or $\operatorname{MRSE}(P=0.23)$. 


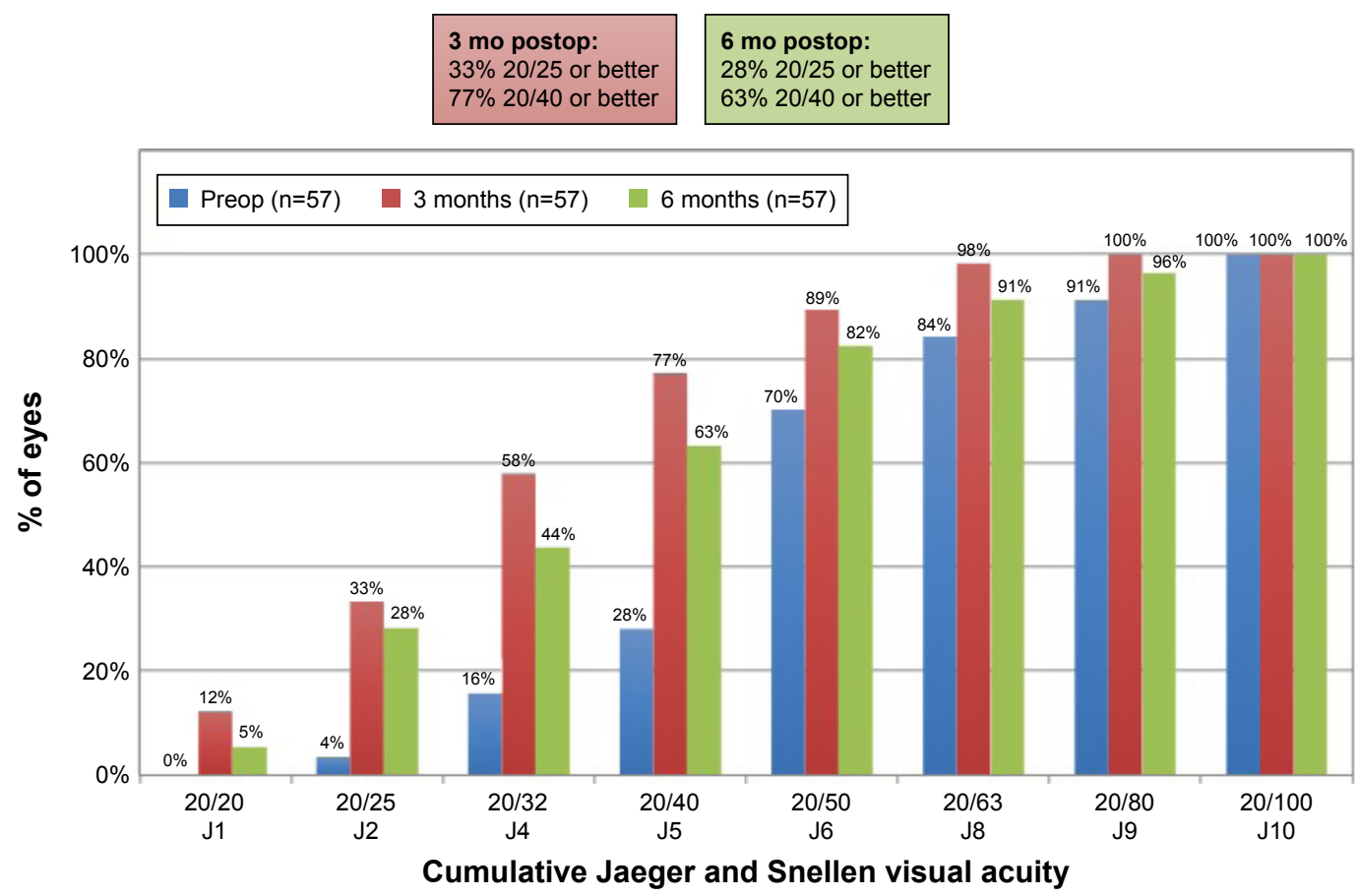

Figure I Cumulative monocular UNVA shown before KAMRA inlay implantation and at 3 and 6 months postoperatively.

Note: The Jaeger (J) acuity scale ranges from JI to J10 or larger, with J2 considered to be the equivalent of 20/20 Snellen visual acuity.

Abbreviations: mo, months; preop, preoperation; postop, postoperation; UNVA, uncorrected near visual acuity.

The postoperative UNVA at 6 months based on pocket depth is shown in Figure 4. The deeper pocket group had better UNVA than the shallow group $(P=0.02)$. At 6 months postoperatively, $71 \%(5 / 7)$ of patients had $20 / 25$ vision or better in the deep pocket group, whereas only $22 \%(11 / 50)$ of patients had 20/25 or better in the shallow pocket group.
At 6 months postoperatively, the mean MRSE in the shallow pocket group was $+0.02 \pm 0.85 \mathrm{SD}$ with a mean change of $+0.52 \pm 0.75$. The MRSE in the deep pocket group was $-0.95 \pm 0.65 \mathrm{SD}$ with a mean change of $-0.30 \pm 0.37 \mathrm{SD}$. The difference in MRSE between the shallow pocket and deep pocket group is statistically significant $(P=0.01)$. In the

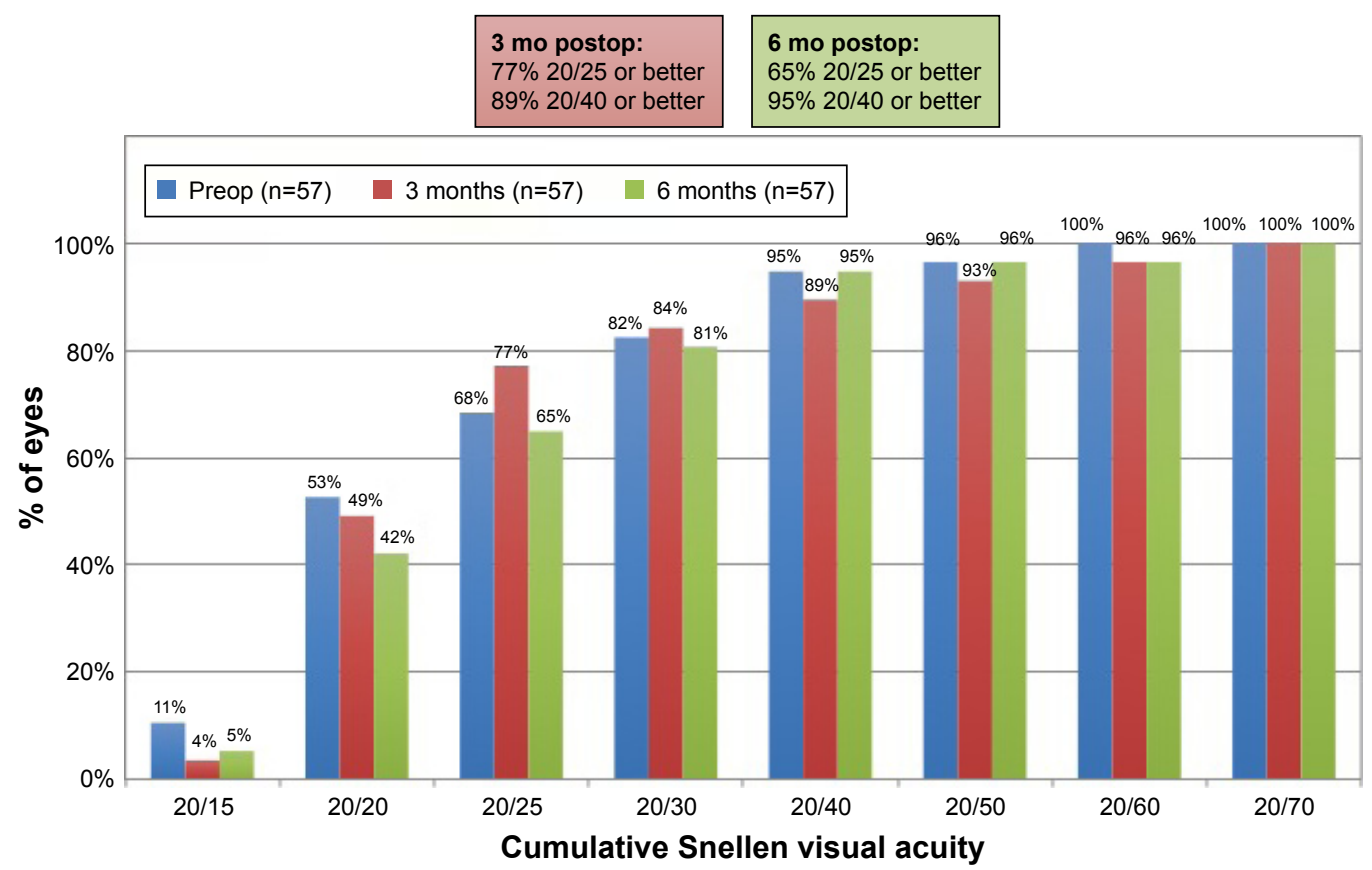

Figure 2 Cumulative monocular UDVA shown before KAMRA inlay implantation and at 3 and 6 months postoperatively. Abbreviations: mo, months; preop, preoperatively; postop, postoperatively; UDVA, uncorrected distance visual acuity. 


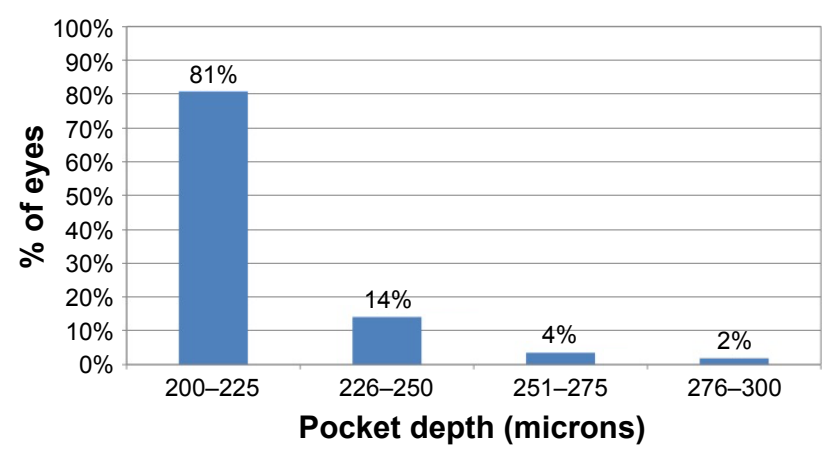

Figure 3 Corneal pocket depth distribution.

shallow pocket group 36\% (18/50) of patients had a hyperopic shift of $\geq+0.75$ and $0 \%(0 / 7)$ of patients in the deep group had a hyperopic shift of $\geq+0.75$.

\section{Virgin and post-LASIK comparison}

The postoperative change in UNVA at 6 months in the post-LASIK group was $-0.17 \log$ MAR $\pm 0.13 \mathrm{SD}$. The postoperative change in UNVA at 6 months in the virgin eye group was $-0.11 \operatorname{logMAR} \pm 0.16 \mathrm{SD}$. There was no significant difference in the change of UNVA between these two groups $(P=0.21)$.

\section{Safety and complications}

The preoperative and postoperative CDVA is shown in Figure 5. At 3 and 6 months postoperatively, the mean CDVA was $20 / 20 \pm 0.03$ and $20 / 20 \pm 0.04$, respectively. The change in Snellen lines after surgery is shown in Figure 6.
No patients lost $>1$ line and $81 \%(45 / 57)$ of patients had no change at 6 months.

The KAMRA inlay was repositioned in five patients. Changes in UNVA following recentration are shown in Figure 7. UNVA improved after repositioning with no change in UDVA.

Only one patient underwent explantation. The inlay was removed at 10 months postoperatively due to dissatisfaction with visual acuity. One month after explantation, the patient successfully returned to baseline with a UNVA of J3 (20/30), UDVA of 20/15, and CDVA of 20/15.

\section{Patient satisfaction}

Of the 57 patients with 3 and 6 months follow-up, 50 patients completed postoperative questionnaires regarding satisfaction. At 3 months postoperatively, 36 (72\%) patients reported some level of satisfaction, eleven of which were "very satisfied." At 6 months, 30 (60\%) patients reported some level of satisfaction, eight of which were "very satisfied". The overall patient satisfaction at 6 months postoperatively is shown in Figure 8.

At 3 months postoperatively, 18 (36\%) patients reported being "not dependent" on reading glasses and two (4\%) patients reported being "always dependent". At 6 months postoperatively, $13(26 \%)$ patients reported being "not dependent" and eight (16\%) patients reported being "always dependent". The rate dependency on reading glasses 6 months postoperatively is shown in Figure 9.

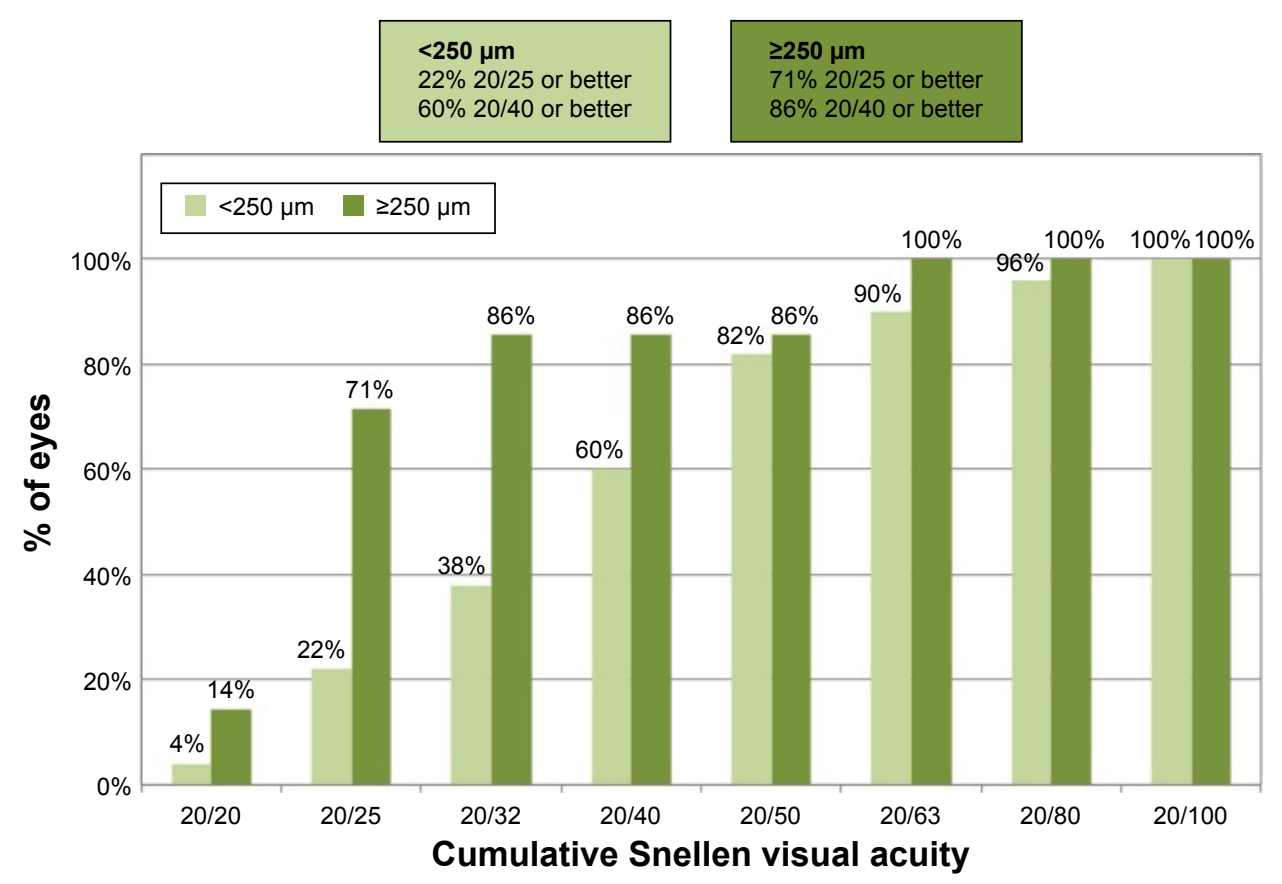

Figure 4 Cumulative monocular UNVA for the deep and shallow pocket groups shown before KAMRA inlay implantation and at 6 months postoperatively. Abbreviation: UNVA, uncorrected near visual acuity. 


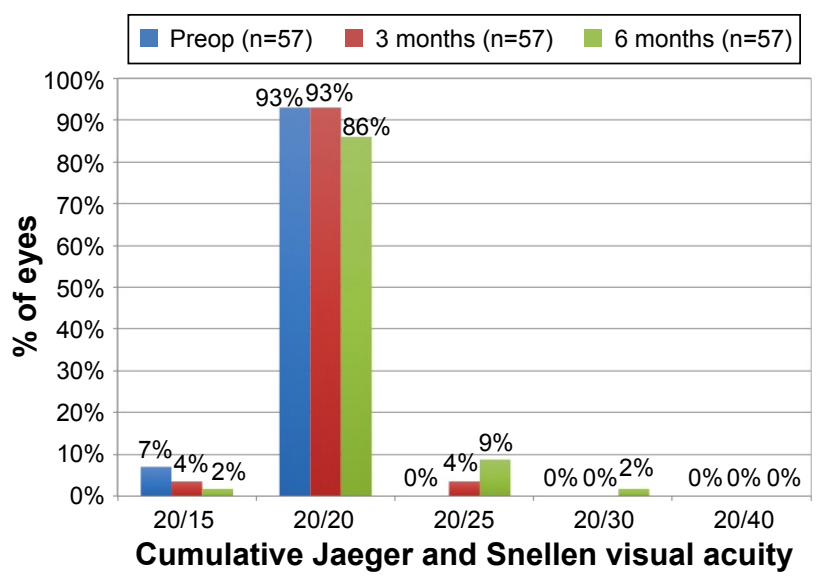

Figure 5 Monocular CDVA shown before KAMRA inlay implantation and at 3 and 6 months postoperatively.

Abbreviations: CDVA, corrected distance visual acuity; preop, preoperatively.

In response to the question "Are you able to do most $(>75 \%)$ things without reading glasses in good light?" $38(76 \%)$ patients said yes at 3 months and $31(62 \%)$ said yes at 6 months.

\section{Discussion}

The KAMRA corneal inlay has been shown to be an effective treatment for the correction of UNVA in presbyopic patients. ${ }^{5-9}$ Our patients achieved a UNVA of 20/40 or better in $77 \%$ and $63 \%$ of eyes at 3 and 6 months, respectively. The primary effectiveness criterion for the KAMRA corneal inlay, as established by the FDA clinical trials, is a monocular UNVA of $20 / 40$ or better in $75 \%$ of eyes 12 months after surgery, of which they achieved $83.5 \%(n=478) .{ }^{12}$ Our slightly differing outcomes compared to the FDA clinical trials were thought to be attributable to our inclusion of postLASIK patients in our visual outcome analysis. Notably, the

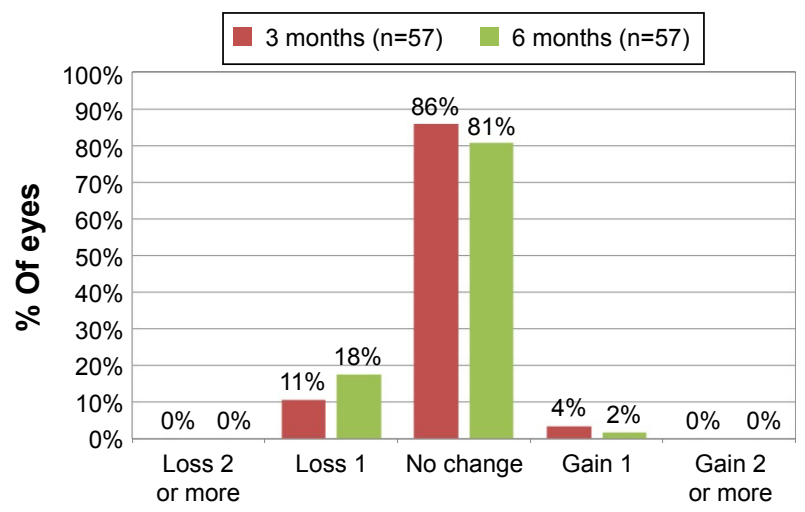

Change in Snellen lines of CDVA

Figure 6 Changes in Snellen lines for monocular CDVA shown before KAMRA inlay implantation and at 3 and 6 months postoperatively.

Abbreviation: CDVA, corrected distance visual acuity.

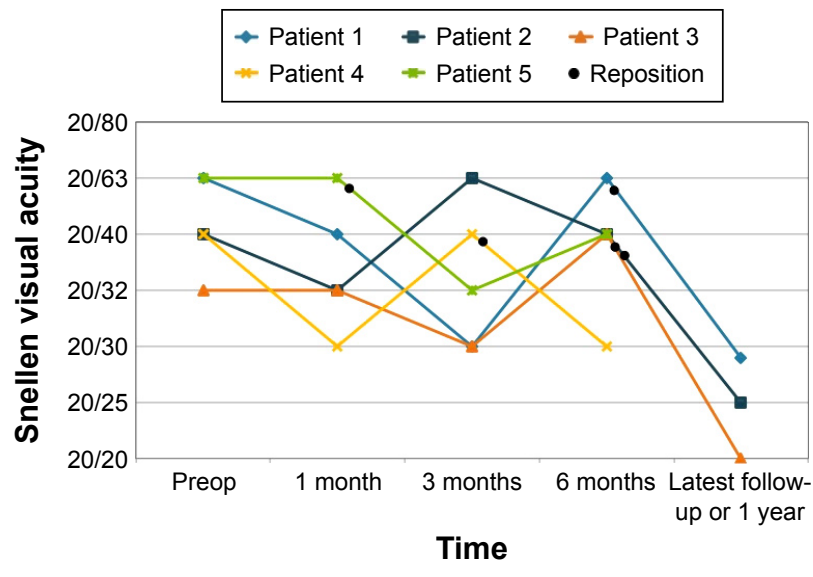

Figure 7 Changes in monocular UNVA in patients who undergo recentering of KAMRA inlay. Black dots represent approximate time that patient underwent recentering. Abbreviations: preop, preoperatively; UNVA, uncorrected near visual acuity.

FDA clinical trials did not include these patients. However, we show that there was no significant change in UNVA at 6 months between our virgin eye and post-LASIK eye groups. Other possible explanations for the differences between our data and FDA clinical trials may be because patients who underwent explantation and recentration, unlike the FDA clinical trials, were included. ${ }^{13}$ Although the percent of patients achieving a monocular UNVA of 20/40 or better was suboptimal, binocular UNVA was 77\%. Although our group characteristics may have negatively impacted postoperative visual acuity, it is important to include these patients to provide data that are more representative of the actual patient population in most refractive surgery practices.

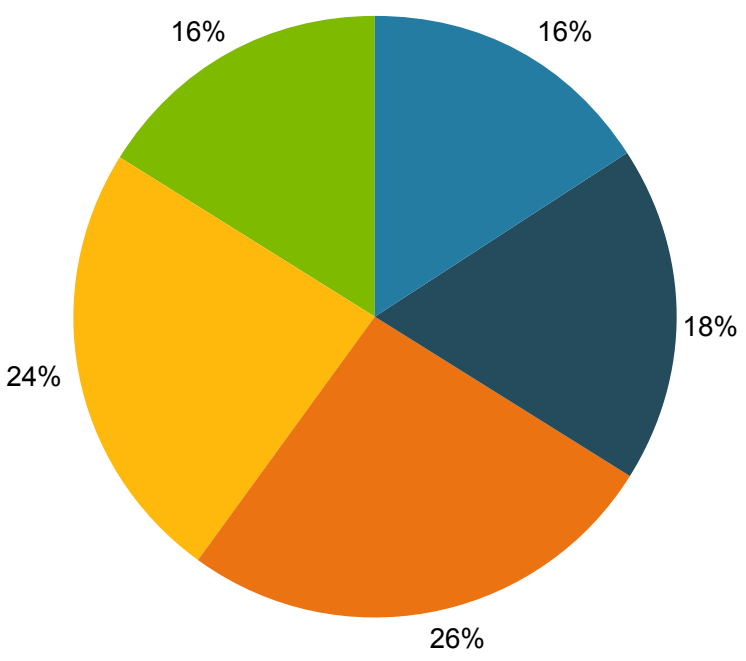
Very satisfied $(n=8)$
Satisfied $(n=9)$
Somewhat satisfied $(n=13)$ Somewhat dissatisfied $(n=12)$ Dissatisfied $(n=8)$

Figure 8 Overall patient satisfaction at 6 months postoperatively. 


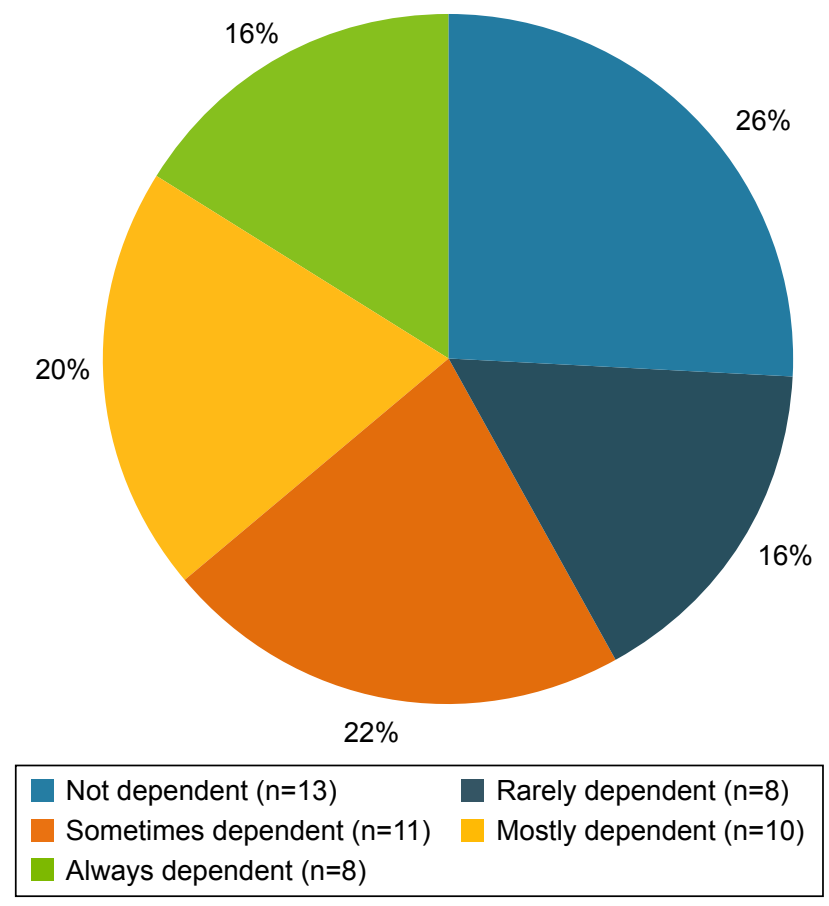

Figure 9 Rate dependency on reading glasses at 6 months postoperatively.

Similar to FDA reports, no significant change in UDVA and CDVA following KAMRA inlay was reported. In our study, $18 \%$ of patients lost 1 line of vision at 6 months postoperatively. This was likely due to ocular surface dryness with secondary mild irregular astigmatism.

With surgical experience, it was found that a deeper pocket depth may produce better postoperative visual outcomes. Our results suggest that a deeper pocket depth is associated with better postoperative UNVA at 6 months. The MRSEs of our deep and shallow pocket groups were also compared. There was a statistically significant difference between groups, with the shallow pocket group having a greater hyperopic shift. None of our patients in the deep pocket group showed a hyperopic shift $>+0.75$ (greatest was +0.13 ), but $36 \%$ of our patients in the shallow group did. It has been speculated by other clinicians that placement of the KAMRA inlay in deeper dissection planes may reduce the inflammatory cascade in terms of recruitment of keratocytes to the site of implantation and stromal remodeling. There is also a belief that when the KAMRA inlay was placed under the thinner LASIK flaps in the past, it induced epithelial changes that led to a hyperopic shift. ${ }^{14}$

Inlay recentration rate reported in the FDA clinical trials was $1(0.2 \%)$ at 12 months, $6(1.2 \%)$ at 24 months, and $6(1.2 \%)$ at 36 months. These patients showed an improvement in UNVA without a significant change in UDVA. Nine percent of our patients underwent KAMRA inlay recentration. All patients had improved UNVA following recentration without a significant change in UDVA. The most common reasons for repositioning the inlay were dissatisfaction with postoperative UNVA and poor inlay positioning as determined by subjective evaluation.

One advantage of the KAMRA inlay is its removability. When the inlay is removed, the patient's visual acuity returns to ranges comparable to the preoperative state. ${ }^{11,15,16}$ In the FDA clinical trials, $15(3 \%)$ patients had their inlay removed at 12 months, $36(7 \%)$ at 24 months, and $44(8 \%)$ at 36 months. The majority of inlay removals $(86 \%)$ were for visual reasons with the most common reason being hyperopic shift. Only one (2\%) patient in our study had undergone inlay explantation by 6-month follow-up, due to dissatisfaction regarding visual acuity. Similar to FDA results, the visual acuity of this patient returned to baseline after explantation.

Patient satisfaction after KAMRA inlay has been evaluated by several studies and has been shown to be good. ${ }^{7,911,17}$ Patient satisfaction from the FDA clinical trials was not reported. Our results show that a majority of patients report some level of satisfaction with their KAMRA inlay. Despite a majority of patients reporting overall satisfaction, our results also show a decrease in satisfaction rates between 3 and 6 months. This regression may be the result of UNVA decline as previously discussed. Although patient satisfaction has been addressed in previously published literature, evaluation of patient satisfaction varies by study design, making it challenging to compare with our results.

\section{Limitations}

There are several limitations to our study. Unlike FDA clinical trials, our study is a retrospective chart review and included fewer patients. The learning curve involved in the surgical technique of KAMRA inlay implantation may have negatively impacted our postoperative outcomes.

\section{Conclusion}

The KAMRA inlay is a good option for the surgical correction or presbyopia. Following implantation, patients have improved monocular UNVA and no significant change in monocular UDVA. A deeper pocket depth may be associated with improved UNVA due to a decrease in hyperopic shift. Additional research is necessary with longer follow-up and larger patient populations before any definitive conclusion can be drawn regarding pocket depth and postoperative visual acuity outcomes. Our results suggest no difference in visual outcomes between virgin eyes and post-LASIK eyes. 
Safety rates are high with no significant change in monocular CDVA. Explantation rates and recentration rates are low. Overall, presbyopic patients are satisfied with their postoperative visual acuity following KAMRA inlay implantation.

\section{Acknowledgment}

This study was supported in part by an unrestricted grant from Research to Prevent Blindness, New York, NY, USA.

\section{Disclosure}

The authors report no conflicts of interest in this work.

\section{References}

1. Eye Diseases Prevalence Research Group. The prevalence of refractive errors among adults in the United States, Western Europe, and Australia. Arch Ophthalmol. 2004;122(4):495.

2. Frick KD, Joy SM, Wilson DA, Naidoo KS, Holden BA. The global burden of potential productivity loss from uncorrected presbyopia. Ophthalmology. 2015;122(8):1706-1710.

3. McDonnell PJ, Lee P, Spritzer K, Lindblad AS, Hays RD. Associations of presbyopia with vision-targeted health-related quality of life. Arch Ophthalmol. 2003;121(11):1577-1581.

4. Papadopoulos PA, Papadopoulos AP. Current management of presbyopia. Middle East Afr J Ophthalmol. 2014;21(1):10.

5. Seyeddain O, Riha W, Hohensinn M, Nix G, Dexl AK, Grabner G. Refractive surgical correction of presbyopia with the AcuFocus small aperture corneal inlay: two-year follow-up. J Refract Surg. 2010;26(10): 707-715.

6. Jalali S, der Au WA, Shaarawy T. AcuFocus corneal inlay to correct presbyopia using femto-LASIK. One year results of a prospective cohort study. Klin Monatsbl Augenheilkund. 2016;233(4):360-364.

7. Dexl AK, Jell G, Strohmaier C, et al. Long-term outcomes after monocular corneal inlay implantation for the surgical compensation of presbyopia. J Cataract Refract Surg. 2015;41(3):566-575.
8. Seyeddain O, Bachernegg A, Riha W, et al. Femtosecond laserassisted small-aperture corneal inlay implantation for corneal compensation of presbyopia: two-year follow-up. J Cataract Refract Surg. 2013;39(2):234-241.

9. Dexl AK, Seyeddain O, Riha W, et al. Reading performance and patient satisfaction after corneal inlay implantation for presbyopia correction: two-year follow-up. J Cataract Refract Surg. 2012;38(10): $1808-1816$

10. Dexl AK, Seyeddain O, Riha W, et al. One-year visual outcomes and patient satisfaction after surgical correction of presbyopia with an intracorneal inlay of a new design. J Cataract Refract Surg. 2012;38(2):262-269.

11. Duignan ES, Farrell S, Treacy MP, et al. Corneal inlay implantation complicated by infectious keratitis. Br J Ophthalmol. 2016;100(2): 269-273.

12. AcuFocus: The Small Aperture Company. KAMRA Inlay Professional Use Information. 2015. Available from: http://www.accessdata.fda.gov/ cdrh_docs/pdf12/P120023d.pdf. Accessed June 13, 2016.

13. AcuFocus: The Small Aperture Company. Summary of Safety and Effectiveness Data (SSED). 2015. Available from: http://www. accessdata.fda.gov/cdrh_docs/pdf12/P120023B.pdf. Accessed June 13, 2016.

14. Dupps WJ, Wilson SE. Biomechanics and wound healing in the cornea. Exp Eye Res. 2006;83(4):709-720.

15. Yılmaz ÖF, Alagöz N, Pekel G, et al. Intracorneal inlay to correct presbyopia: long-term results. J Cataract Refract Surg. 2011;37(7): $1275-1281$.

16. Malandrini A, Martone G, Canovetti A, et al. Morphologic study of the cornea by in vivo confocal microscopy and optical coherence tomography after bifocal refractive corneal inlay implantation. J Cataract Refract Surg. 2014;40(4):545-557.

17. Tomita M, Kanamori T, Waring GO, et al. Simultaneous corneal inlay implantation and laser in situ keratomileusis for presbyopia in patients with hyperopia, myopia, or emmetropia: six-month results. $J$ Cataract Refract Surg. 2012;38(3):495-506.
Clinical Ophthalmology

\section{Publish your work in this journal}

Clinical Ophthalmology is an international, peer-reviewed journal covering all subspecialties within ophthalmology. Key topics include: Optometry; Visual science; Pharmacology and drug therapy in eye diseases; Basic Sciences; Primary and Secondary eye care; Patient Safety and Quality of Care Improvements. This journal is indexed on Submit your manuscript here: http://www.dovepress.com/clinical-ophthalmology-journal

\section{Dovepress}

PubMed Central and CAS, and is the official journal of The Society of Clinical Ophthalmology (SCO). The manuscript management system is completely online and includes a very quick and fair peer-review system, which is all easy to use. Visit http://www.dovepress.com/ testimonials.php to read real quotes from published authors. 